Research Paper

\title{
The survival benefit of palliative gastrectomy and/or metastasectomy in gastric cancer patients with synchronous metastasis: a population-based study using propensity score matching and coarsened exact matching
}

Lu-Ping Yang*, Zi-Xian Wang*, Ming-Ming He*, Ying Jin, Chao Ren, Zhi-Qiang Wang, Feng-Hua Wang, Yu-Hong Li, Feng Wang, Rui-Hua Xu ${ }^{\varpi}$

Department of Medical Oncology, Sun Yat-Sen University Cancer Center, State Key Laboratory of Oncology in South China, Collaborative Innovation Center for Cancer Medicine, Guangzhou 510060, China

* These authors contributed equally to this work.

$\triangle$ Corresponding author: Rui-Hua Xu, 651 Dong Feng Road East, Guangzhou 510060, China; Tel and Fax: +86-20-8734 3468; E-mail: xurh@sysucc.org.cn

(c) Ivyspring International Publisher. This is an open access article distributed under the terms of the Creative Commons Attribution (CC BY-NC) license (https://creativecommons.org/licenses/by-nc/4.0/). See http://ivyspring.com/terms for full terms and conditions.

Received: 2018.07.29; Accepted: 2018.12.08; Published: 2019.01.01

\begin{abstract}
Introduction: Palliative surgeries were controversial for asymptomatic metastatic gastric cancer (mGC) patients. This study was aimed to evaluate survival benefit of palliative surgeries to gastric and/or metastatic tumors in $\mathrm{mGC}$ patients based on U.S population.

Materials and Methods: A total of 8345 gastric cancer patients diagnosed with synchronous distal metastasis between 2004 to 2013 from the Surveillance, Epidemiology, and End Results Program (SEER) database were divided into four groups according to surgery strategies: surgeries to both primary and metastatic tumors (SPM), gastrectomy only (GO), metastasectomy only (MO) and no surgery performed (NS). Their clinicopathological characteristics and overall survival (OS) were analyzed before and after propensity score matching (PSM) and coarsened exact matching (CEM).

Results: The median OS of SPM and GO patients was both significantly higher than NS patients (11 months vs. 8 months vs. 5 months; $P<0.001$, respectively) while that of $M O$ was not $(6$ months vs. 5 months; $P=0.286)$. In comparisons between surgery strategies, survival benefit was similar between SPM and GO groups $(P=0.389)$ and both showed significantly better survival than $M O$ patients $(P<0.001)$. All surgery strategies were proved to be favorable prognostic factors over non-surgical treatment (Hazard ratio (HR) for SPM: 0.60, $P<0.001$; HR for GO: 0.62, $P<0.001$; HR for MO: 0.91, $P=0.046$ ). Similar results were obtained after matching by PSM and CEM except that prognostic impact of $M O$ deteriorated.

Conclusions: Gastrectomy plus metastasectomy or gastrectomy alone could be adopted as a choice of improving survival in the U.S population. Metastasectomy alone is not generally recommended.
\end{abstract}

Key words: gastric cancer; gastrectomy; metastasectomy; survival; matching

\section{Introduction}

Gastric cancer (GC) ranks the fifth most common tumors and the fourth leading cause of cancer-related deaths in the United States, with approximately 22,220 new cases and 10,990 deaths in 2014. [1] Although an annual decline of the incidence rate of GC was observed since 1992 [2], the 5-year overall survival (OS) remained unimproved. Especially for metastatic GC (mGC), the median survival was only 7.9 to 13.8 months as assessed in several randomized clinical trials, which however consisted of more than one third of initially diagnosed GC patients. [3] Developing best treatment strategies for mGC is 
undoubtedly of vital importance. Systemic treatment strategies with chemotherapy, target therapy and immunotherapy are universally adopted while performing surgery is controversial. Current guidelines approved of palliative gastrectomy in terms of obstruction or uncontrolled bleeding, but palliative surgeries for the purpose of tumor reduction to either primary or metastatic sites were not mentioned for recommendation. [4] There were some studies finding that surgical resection with therapeutic intent in mGC patients was associated with a relatively poor prognosis, and the REGATTA trial conducted in Eastern population negated gastrectomy followed by chemotherapy could improve survival in mGC patients compared to chemotherapy alone. [5, 6] Nevertheless, in recent years many retrospective studies challenged the negative results and have shown survival benefit in patients treated with gastrectomy and/or metastasectomy with or without chemotherapy. [7-14] Moreover, the GYMSSA trial proved regional treatment including maximal cytoreductive surgery and regional hyperthermic intraperitoneal chemotherapy combined with systemic chemotherapy could achieve prolonged survival in selected patients. [15] For now, the survival impacts of surgeries to mGC patients in Western population are still under investigation, and there also lacks evidence in comparing different surgery strategies.

In this study, we searched the Surveillance, Epidemiology, and End Results Program (SEER) database to evaluate survival benefit from surgeries to primary and/or metastatic tumors in comparison with non-surgeryin the U.S. population.

\section{Material and methods}

\subsection{Patients and data collection}

The study population and their clinicopathological data were searched and collected from Surveillance, Epidemiology, and End Results Program (SEER) database. Patients initially diagnosed as gastric adenocarcinoma by microscopically confirmation with synchronous distal metastasis between 2004 to 2013 at the age of 18 to 79 were included. GC was defined according to the International Classification of Diseases for Oncology, Third Edition (ICD-O-3) with codes C160/C161/C162/C163/C164/C165/C166/C168/C1 69 for primary tumor location and codes $8140 / 8144 / 8145 / 8211 / 8260 / 8480 / 8490$ for histological type. Patients aged 80 or more are excluded because they are not usually considered for surgery and thus not fit in this topic. Patients diagnosed with more than one primary tumor and patients undergoing radiation therapy were excluded to avoid distraction from study objectives. It was necessary to clarify each patient's surgery strategy that whether one underwent primary or metastatic tumor resection, and thus those without such information would also be excluded. Additionally, patients who died within one month after diagnosis were excluded for more sufficient analysis. Figure 1 presents the inclusion and exclusion process. A total of 8345 eligible patients were divided into four groups according to the surgery strategy, that is one group of patients who underwent surgeries to both primary and metastatic tumors (SPM), one group with gastrectomy only (GO), one group with metastasectomy only (MO) and one group of the other patients who was not surgically operated (NS).

No institutional approval or informed consent was required because SEER is a public-use database.

\subsection{Statistical analysis}

Patient characteristics were compared by the chi-square test for categorical variables. Spearman rank correlation analysis was used to evaluate time trends of surgery strategies. Overall survival (OS) was the primary endpoint outcome and was evaluated by Kaplan-Meier method. Log-rank test was used to compare survival between groups. To analyze prognostic factors, the univariable Cox regression analysis was performed and hazard ratio (HR) with 95\% confidence interval (CI) were assessed, followed by a multivariable Cox analysis to evaluate prognostic impact of palliative surgeries with risk adjustment for the other factors whose $P$ value $<0.05$ in the univariable Cox analysis. Furthermore, two matching methods were introduced to guarantee better balance in baseline characteristics between groups and to reduce confounding impacts on survival analysis. One matched subanalysis was based on the propensity score with 1-to-1-to-1-to-1 matching (PSM). [16] Coarsened exact matching (CEM) method, which is less fitting-model dependent than PSM and could improve balance for each covariate without influencing the others to achieve maximum of the balance, was used in the other subanalysis. [17-19] Population after matching was analyzed as it was before matching.

Information on chemotherapy in the SEER database was limited to patients with or without/unknown receiving chemotherapy, and was suggested to be used with cautions due to its incompleteness and potential biases according to the official data use agreement. However, systemic chemotherapy is a major treatment for mGC patients and have a strong impact on their survival, therefore we carried out sensitivity analyses to verify the 
survival benefit of surgery strategies in combination of chemotherapy after excluding patients receiving no/unknown chemotherapy.

All data analyses were performed using SPSS Statistics 22.0 (IBM Corp., Version 22.0, Armonk, NY) and the "MatchIt" and "cem" R packages (The R Foundation, version 3.4.2). A two-sided $P$ value $<0.05$ was considered to be statistically significant.

\section{Results}

\subsection{Patient characteristics}

Table 1 shows the demographic and clinicopathological characteristics of the patients enrolled. The median age of the whole cohort was 61 years old and $62.3 \%$ was male. Non-Hispanic white people and west-region residents took the most proportion, respectively.

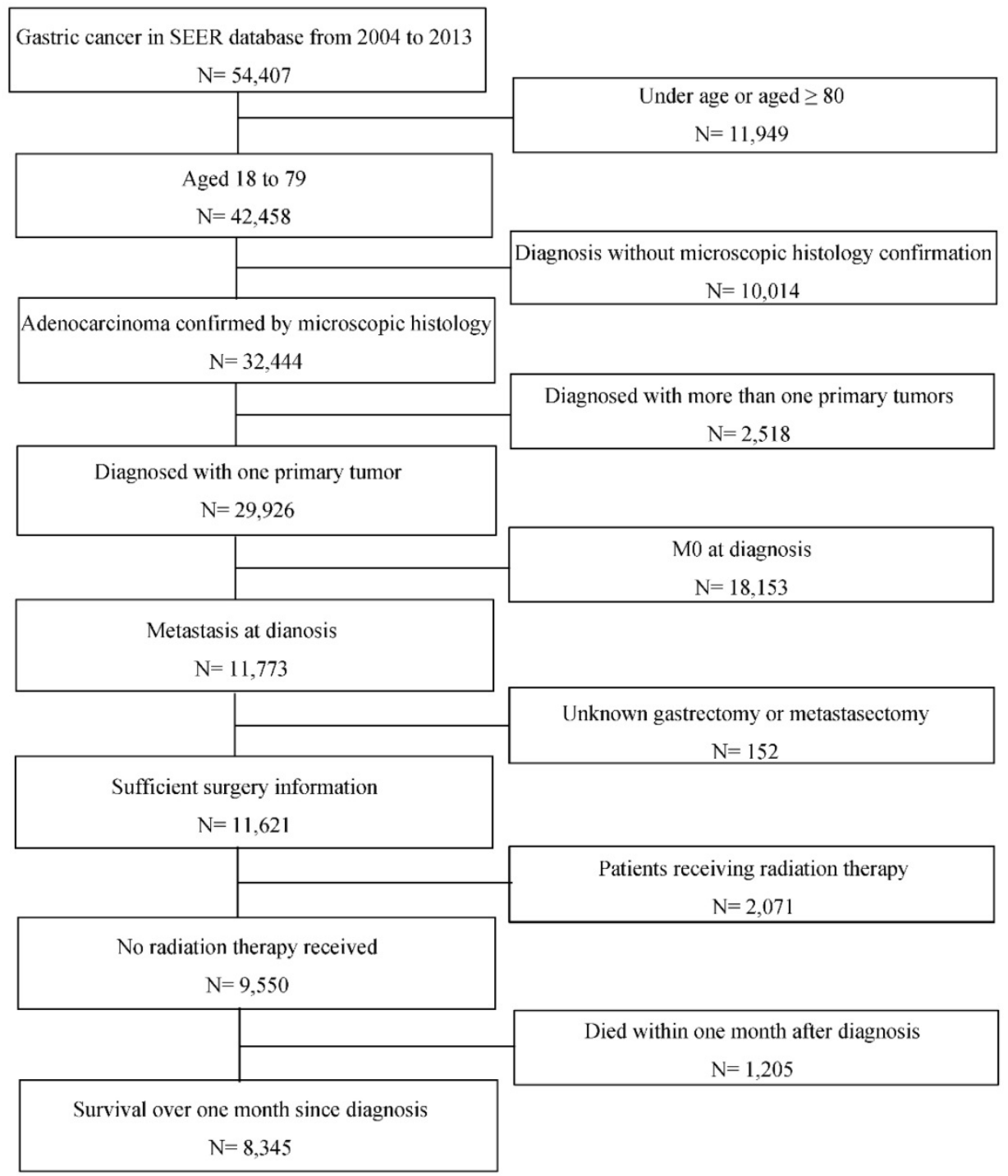

Figure 1. Flowchart of inclusion and exclusion process of the study population. Abbreviations: SEER, Surveillance, Epidemiology, and End Results; M0, without distant metastasis diagnosed.

Seventy-seven percent of patients had no surgery, followed by $12.1 \%$ of patients with gastrectomy only and $6.6 \%$ with metastatic tumors resected only, and smallest proportion of patients underwent SPM. Imbalance between groups was found in all characteristics investigated. In SPM and $\mathrm{MO}$ groups, there were more younger patients $(<50$ years old) and female than that in other groups. NS patients had more primary tumor located in the upper one third whereas SPM and GO patients had more in the lower one third stomach. Besides, more and more patients received non-surgical treatment and an over-ten-percent increase was observed from 2004 to 2013 while the rate of mGC patients undergoing gastrectomy dropped significantly as time went by. $(P<0.001$, Figure 2) 
Table 1. Baseline demographic and clinicopathological characteristics of the patients

\begin{tabular}{|c|c|c|c|c|c|c|}
\hline \multirow[t]{2}{*}{ Variable } & \multicolumn{5}{|l|}{ Number (\%) } & \multirow[t]{2}{*}{$P$ value } \\
\hline & All cohort & SPM & GO & MO & NS & \\
\hline Total & $8345(100.0 \%)$ & $359(4.3 \%)$ & $1006(12.1 \%)$ & $551(6.6 \%)$ & $6429(77.0 \%)$ & \\
\hline Age, years & & & & & & $<0.001$ \\
\hline$<50$ & $1819(21.8 \%)$ & $106(29.5 \%)$ & $207(20.6 \%)$ & $206(37.4 \%)$ & $1300(20.2 \%)$ & \\
\hline $50-59$ & $2028(24.3 \%)$ & $87(24.2 \%)$ & $254(25.2 \%)$ & $138(25.0 \%)$ & $1549(24.1 \%)$ & \\
\hline $60-69$ & $2449(29.3 \%)$ & $83(23.1 \%)$ & $297(29.5 \%)$ & $120(21.8 \%)$ & $1949(30.3 \%)$ & \\
\hline$\geq 70$ & $2049(24.6 \%)$ & $83(23.1 \%)$ & $248(24.7 \%)$ & $87(15.8 \%)$ & $1631(25.4 \%)$ & \\
\hline Sex & & & & & & $<0.001$ \\
\hline Male & $5195(62.3 \%)$ & $179(49.9 \%)$ & $629(62.9 \%)$ & $212(38.5 \%)$ & 4175 (64.9\%) & \\
\hline Female & $3150(37.7 \%)$ & $180(50.1 \%)$ & $377(37.5 \%)$ & $339(61.5 \%)$ & $2254(35.1 \%)$ & \\
\hline Marriage status & & & & & & 0.012 \\
\hline Married & $5005(60.0 \%)$ & $227(63.2 \%)$ & $631(62.7 \%)$ & $327(59.3 \%)$ & $3820(59.4 \%)$ & \\
\hline Widowed & $670(8.0 \%)$ & $28(7.8 \%)$ & $97(9.6 \%)$ & $35(6.4 \%)$ & $510(7.9 \%)$ & \\
\hline Other & $2670(32.0 \%)$ & $104(29.0 \%)$ & $278(27.6 \%)$ & $189(34.3 \%)$ & $2099(32.6 \%)$ & \\
\hline Race & & & & & & $<0.001$ \\
\hline Non-Hispanic White & $3973(47.6 \%)$ & $170(47.4 \%)$ & $389(38.7 \%)$ & $242(43.9 \%)$ & $3172(49.3 \%)$ & \\
\hline Non-Hispanic Black & $1123(13.5 \%)$ & $42(11.7 \%)$ & $158(15.7 \%)$ & $67(12.2 \%)$ & $856(13.3 \%)$ & \\
\hline Hispanic & $2030(24.3 \%)$ & $93(25.9 \%)$ & $271(26.9 \%)$ & $151(27.4 \%)$ & $1515(23.6 \%)$ & \\
\hline Other & $1219(14.6 \%)$ & $54(15.0 \%)$ & $188(18.7 \%)$ & $91(16.5 \%)$ & $886(13.8 \%)$ & \\
\hline SEER region & & & & & & $<0.001$ \\
\hline Mid-west & $917(11.0 \%)$ & $43(12.0 \%)$ & $69(6.9 \%)$ & $71(12.9 \%)$ & $734(11.4 \%)$ & \\
\hline Northeast & $1327(15.9 \%)$ & $62(17.3 \%)$ & $143(14.2 \%)$ & $71(12.9 \%)$ & $1051(16.3 \%)$ & \\
\hline South & $1339(16.0 \%)$ & $67(18.7 \%)$ & $162(16.1 \%)$ & $85(15.4 \%)$ & $1025(15.9 \%)$ & \\
\hline West & $4762(57.1 \%)$ & $187(52.1 \%)$ & $632(62.8 \%)$ & $324(58.8 \%)$ & $3619(56.3 \%)$ & \\
\hline Year of diagnosis & & & & & & $<0.001$ \\
\hline 2004-2006 & $2387(28.6 \%)$ & $130(36.2 \%)$ & $389(38.7 \%)$ & $179(32.5 \%)$ & $1689(26.3 \%)$ & \\
\hline 2007-2009 & $2460(29.5 \%)$ & $114(31.8 \%)$ & $285(28.3 \%)$ & $145(26.3 \%)$ & $1916(29.8 \%)$ & \\
\hline 2010-2013 & $3498(41.9 \%)$ & $115(32.0 \%)$ & $332(33.0 \%)$ & $227(41.2 \%)$ & $2824(43.9 \%)$ & \\
\hline Primary tumor location & & & & & & $<0.001$ \\
\hline Upper one third & $2537(30.4 \%)$ & $52(14.5 \%)$ & $138(13.7 \%)$ & $105(19.1 \%)$ & $2242(34.9 \%)$ & \\
\hline Middle one third & $841(10.1 \%)$ & $35(9.7 \%)$ & $93(9.2 \%)$ & $64(11.6 \%)$ & $649(10.1 \%)$ & \\
\hline Lower one third & $1568(18.8 \%)$ & $105(29.2 \%)$ & $333(33.1 \%)$ & $99(18.0 \%)$ & $1031(16.0 \%)$ & \\
\hline NOS & $3399(40.7 \%)$ & $167(46.5 \%)$ & $442(43.9 \%)$ & $283(51.4 \%)$ & $2507(39.0 \%)$ & \\
\hline Tumor grade & & & & & & $<0.001$ \\
\hline $\mathrm{G} 1 / \mathrm{G} 2$ & $1484(17.8 \%)$ & $56(15.6 \%)$ & $177(17.6 \%)$ & $46(8.3 \%)$ & $1205(18.7 \%)$ & \\
\hline G3/G4 & $5121(61.4 \%)$ & $286(79.7 \%)$ & $764(75.9 \%)$ & $310(56.3 \%)$ & $3761(58.5 \%)$ & \\
\hline Unknown & $1740(20.9 \%)$ & $17(4.7 \%)$ & $65(6.5 \%)$ & $195(35.4 \%)$ & $1463(22.8 \%)$ & \\
\hline Histology & & & & & & $<0.001$ \\
\hline Adenocarcinoma NOS & $5774(69.2 \%)$ & $219(61.0 \%)$ & $628(62.4 \%)$ & $270(49.0 \%)$ & $4657(72.4 \%)$ & \\
\hline Mucinous adenocarcinoma & $135(1.6 \%)$ & $9(2.5 \%)$ & $31(3.1 \%)$ & $10(1.8 \%)$ & $85(1.3 \%)$ & \\
\hline Signet ring cell carcinoma & $2436(29.2 \%)$ & $131(36.5 \%)$ & $347(34.5 \%)$ & $271(49.2 \%)$ & $1687(26.2 \%)$ & \\
\hline \multicolumn{7}{|l|}{ Gastrectomy } \\
\hline Yes & $1365(16.4 \%)$ & $359(100.0 \%)$ & $1006(100.0 \%)$ & $0(0.0 \%)$ & $0(0.0 \%)$ & \\
\hline Total gastrectomy surgery & $401(4.8 \%)$ & $115(32.0 \%)$ & $286(28.4 \%)$ & $0(0.0 \%)$ & $0(0.0 \%)$ & \\
\hline Non-total gastrectomy surgery & $907(10.9 \%)$ & $216(60.2 \%)$ & $691(68.7 \%)$ & $0(0.0 \%)$ & $0(0.0 \%)$ & \\
\hline Not specified & $57(0.7 \%)$ & $28(7.8 \%)$ & $29(2.9 \%)$ & $0(0.0 \%)$ & $0(0.0 \%)$ & \\
\hline No & $6980(83.6 \%)$ & $0(0.0 \%)$ & $0(0.0 \%)$ & $551(100.0 \%)$ & $6429(100.0 \%)$ & \\
\hline \multicolumn{7}{|l|}{ Metastasectomy } \\
\hline Yes & $910(10.9 \%)$ & $359(100.0 \%)$ & $0(0.0 \%)$ & $551(100.0 \%)$ & $0(0.0 \%)$ & \\
\hline No & $7435(89.1 \%)$ & $0(0.0 \%)$ & $1006(100.0 \%)$ & $0(0.0 \%)$ & $6429(100.0 \%)$ & \\
\hline
\end{tabular}

Abbreviation: SPM, surgeries to both primary and metastatic tumors; GO, gastrectomy only; MO, metastasectomy only; NS, no surgery; SEER, Surveillance, Epidemiology, and End Results.

\subsection{Survival analysis}

The OS of SPM and GO patients was both significantly higher than NS patients, with an improved median OS (mOS) of 6.0 months and 3.0 months, respectively. However, the mOS of MO only exceeded that of NS by 1.0 month, which was not a significant difference $(P=0.286)$. In terms of comparisons between surgery strategies, survival benefit was similar between SPM and GO groups $(P=0.389)$, while both showed significantly better survival than $\mathrm{MO}$ patients $(P<0.001$, respectively). Figure 3 depicts survival curves of patients stratified by surgical strategies.

\subsection{Univariable and multivariable Cox regression analysis}

Age at diagnosis, marriage status, race, SEER region, location and histology of primary tumor, year of diagnosis and surgery strategies were proven to be independent prognostic factors (Table 2). After adjusting for these factors in multivariable Cox analysis, all surgery strategies were proved to have significantly better prognosis than NS ones (HR for SPM: 0.60, $P<0.001$; HR for GO: 0.62, $P<0.001$; HR for MO: 0.91, $P=0.046$ ), and gastrectomy with or without metastasectomy remained superior over $\mathrm{MO}$. 
$100.0 \%$
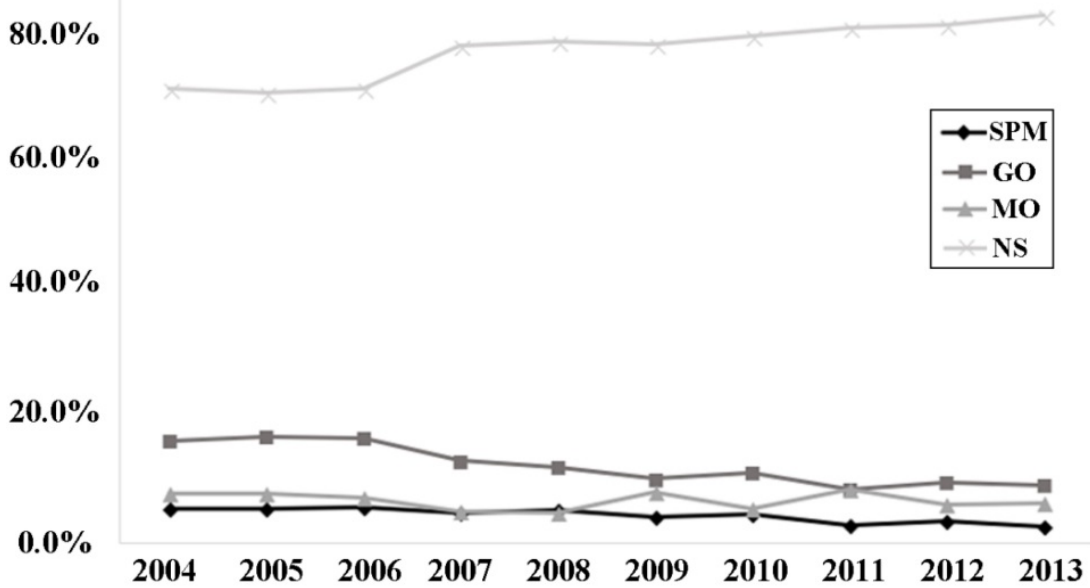

Figure 2. Proportions of surgery strategies performed in each year from 2004 to 2013 . Abbreviations: SPM, surgeries to both primary and metastatic tumors; GO, gastrectomy only; MO, metastasectomy only; NS, no surgery.

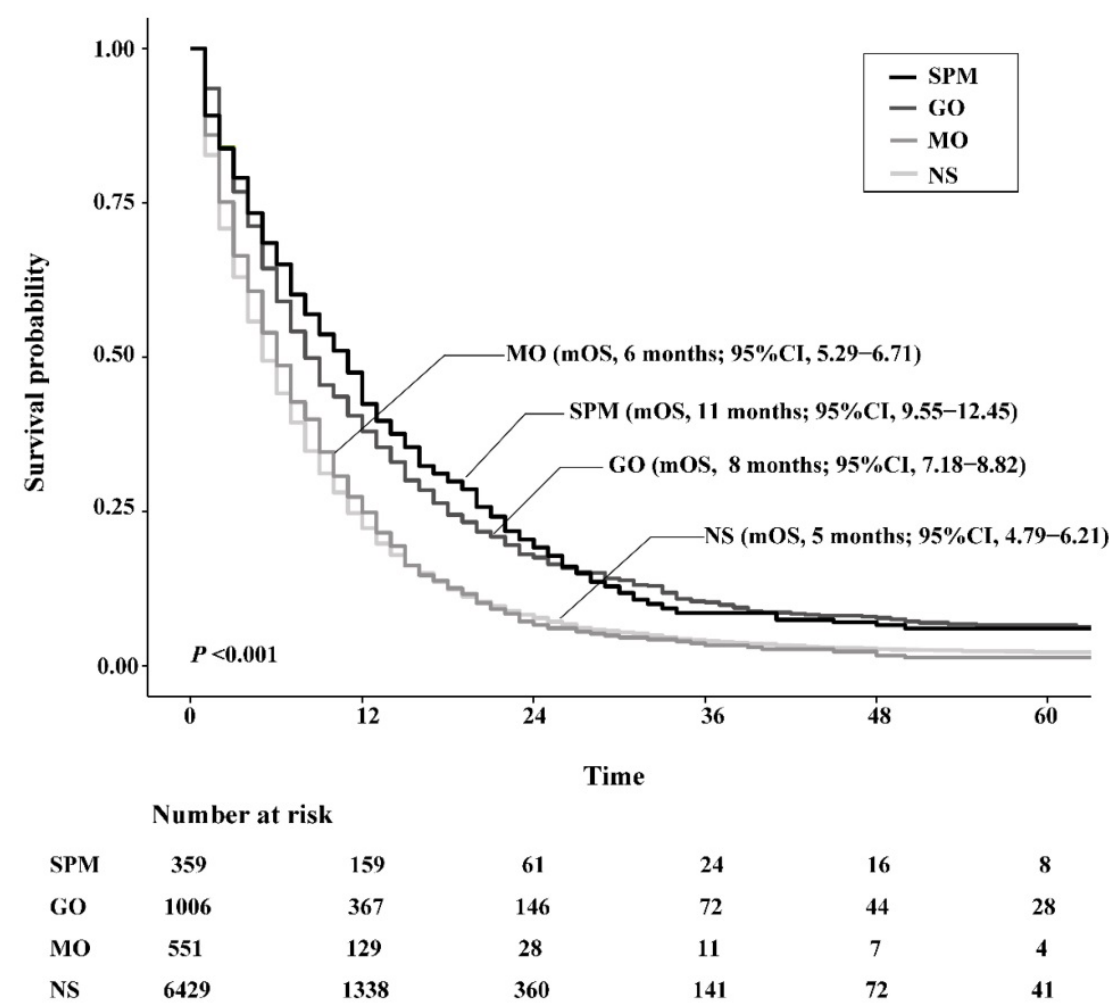

Figure 3. Kaplan-Meier survival curves of patients stratified according to their surgery strategies. Abbreviations: SPM, surgeries to both primary and metastatic tumors; GO, gastrectomy only; MO, metastasectomy only; NS, no surgery; mOS, median overall survival.

\subsection{Propensity score matching and coarsened exact matching analysis}

By PSM and CEM, the patient characteristics were well balanced between groups to diminish selection bias (Supplementary Table 1). The survival analyses based upon the matched population both showed consistent results with that upon the unmatched cohort, except in CEM-weighted analysis MO group presented poorer yet not significant survival than NS group (Figure 4).
In the univariable and multivariable Cox analysis, surgery strategies were demonstrated to be a prognostic factor (Table 3). Surgeries to the primary and/or metastatic tumors lowered the death risk from non-surgery, however MO did not function significantly in both matched subanalyses. Inbetween surgery strategies, SPM and GO held comparable survival impacts and both were more favorable than MO. 
Table 2. Results of univariable and multivariable Cox regression analysis

\begin{tabular}{|c|c|c|c|c|}
\hline \multirow[t]{2}{*}{ Variable } & \multicolumn{2}{|c|}{ Univariable Cox analysis } & \multicolumn{2}{|c|}{$\begin{array}{l}\text { Multivariable Cox } \\
\text { analysis }\end{array}$} \\
\hline & HR (95\% CI) & $P$ value & HR $(95 \%$ CI) & $P$ value \\
\hline \multicolumn{5}{|l|}{ Age, years } \\
\hline$<50$ & Reference & $<0.001$ & Reference & $<0.001$ \\
\hline $50-59$ & $0.97(0.91,1.04)$ & & $0.99(0.93,1.07)$ & \\
\hline $60-69$ & $1.05(0.98,1.12)$ & & $1.09(1.02,1.17)$ & \\
\hline$\geq 70$ & $1.22(1.14,1.30)$ & & $1.25(1.16,1.35)$ & \\
\hline \multicolumn{5}{|l|}{ Sex } \\
\hline Male & Reference & 0.309 & $-a$ & - a \\
\hline Female & $1.03(0.98,1.07)$ & & $-a$ & \\
\hline \multicolumn{5}{|l|}{ Marriage status } \\
\hline Widowed & Reference & $<0.001$ & Reference & $<0.001$ \\
\hline Married & $0.79(0.72,0.86)$ & & $0.85(0.77,0.92)$ & \\
\hline Other & $0.86(0.78,0.94)$ & & $0.93(0.85,1.02)$ & \\
\hline \multicolumn{5}{|l|}{ Race } \\
\hline Non-Hispanic White & Reference & 0.005 & Reference & 0.002 \\
\hline Non-Hispanic Black & $1.09(1.02,1.17)$ & & $1.06(0.98,1.15)$ & \\
\hline Hispanic & $1.01(0.96,1.07)$ & & $0.99(0.93,1.06)$ & \\
\hline Other & $0.93(0.87,0.99)$ & & $0.90(0.83,0.96)$ & \\
\hline \multicolumn{5}{|l|}{ SEER region } \\
\hline Mid-west & Reference & $<0.001$ & Reference & $<0.001$ \\
\hline Northeast & $0.83(0.76,0.90)$ & & $0.83(0.76,0.91)$ & \\
\hline South & $1.02(0.93,1.11)$ & & $1.03(0.94,1.13)$ & \\
\hline West & $0.99(0.92,1.07)$ & & $1.02(0.94,1.11)$ & \\
\hline \multicolumn{5}{|l|}{ Year of diagnosis } \\
\hline $2004-2006$ & Reference & $<0.001$ & Reference & $<0.001$ \\
\hline 2007-2009 & $0.86(0.81,0.91)$ & & $0.83(0.78,0.88)$ & \\
\hline 2010-2013 & $0.81(0.76,0.86)$ & & $0.77(0.73,0.82)$ & \\
\hline \multicolumn{5}{|l|}{ Primary tumor location } \\
\hline Upper one third & Reference & $<0.001$ & Reference & $<0.001$ \\
\hline Middle one third & $1.09(1.01,1.18)$ & & $1.08(0.99,1.18)$ & \\
\hline Lower one third & $1.04(0.98,1.12)$ & & $1.11(1.03,1.19)$ & \\
\hline NOS & $1.16(1.10,1.23)$ & & $1.18(1.12,1.26)$ & \\
\hline \multicolumn{5}{|l|}{ Histology } \\
\hline Adenocarcinoma NOS & Reference & 0.001 & Reference & $<0.001$ \\
\hline Mucinous adenocarcinoma & $0.91(0.76,1.10)$ & & $0.95(0.79,1.13)$ & \\
\hline Signet ring cell carcinoma & $1.09(1.04,1.15)$ & & $1.11(1.06,1.18)$ & \\
\hline \multicolumn{5}{|l|}{ Surgery strategies } \\
\hline NS & Reference & $<0.001$ & Reference & $<0.001$ \\
\hline GO & $0.67(0.62,0.72)$ & & $0.62(0.57,0.67)$ & \\
\hline $\mathrm{MO}$ & $0.95(0.87,1.05)$ & & $0.91(0.83,1.00)$ & \\
\hline SPM & $0.63(0.57,0.71)$ & & $0.60(0.54,0.68)$ & \\
\hline
\end{tabular}

a The variable was not include in the multivariable Cox analysis because of its $P$ value $\geq 0.05$ in the univariable Cox analysis.

Abbreviations: HR, hazard ratio; SEER, the Surveillance, Epidemiology, and End Results Program; NS, no surgery; GO, gastrectomy only; MO, metastasectomy only; SPM, surgery to both primary and metastatic tumor; NOS, not otherwise specified.

\section{A}

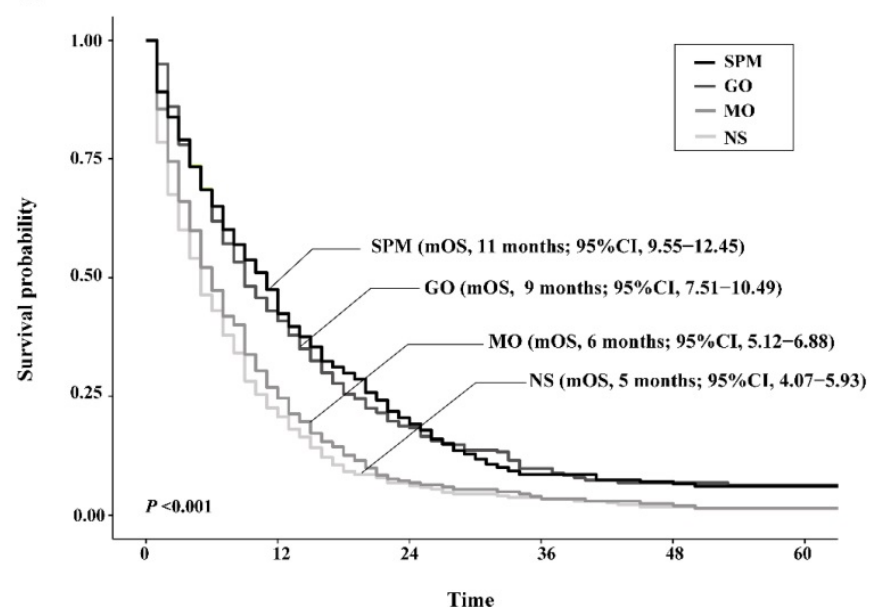

Table 3. Multivariable Cox regression analysis with propensity score matching and coarsened exact matching method weighting

\begin{tabular}{|c|c|c|c|c|}
\hline \multirow[t]{2}{*}{ Surgery strategies } & \multicolumn{2}{|l|}{$\begin{array}{l}\text { Propensity score } \\
\text { matching cohort }\end{array}$} & \multicolumn{2}{|c|}{$\begin{array}{l}\text { Coarsened exact matching method } \\
\text { weighted cohort }\end{array}$} \\
\hline & $\mathrm{HR}^{\mathrm{a}}(\mathbf{9 5 \%} \mathrm{CI})$ & $P$ value & $\mathrm{HR}^{\mathrm{b}}(95 \% \mathrm{CI})$ & $P$ value \\
\hline NS & Reference & & Reference & \\
\hline GO & $0.59(0.51,0.69)$ & $<0.001$ & $0.57(0.43,0.77)$ & $<0.001$ \\
\hline $\mathrm{MO}$ & $0.94(0.80,1.10)$ & 0.412 & $0.89(0.65,1.22)$ & 0.464 \\
\hline SPM & $0.60(0.51,0.70)$ & $<0.001$ & $0.50(0.35,0.72)$ & $<0.001$ \\
\hline \multicolumn{5}{|c|}{$\begin{array}{l}\text { a After adjustment for age, year of diagnosis, primary tumor location and histology } \\
\text { whose } P \text { value }<0.05 \text { in the univariable Cox regression analysis. }\end{array}$} \\
\hline \multicolumn{5}{|c|}{$\begin{array}{l}\text { b After adjustment for age, sex, race, SEER region, year of diagnosis, primary tumor } \\
\text { location and histology whose } P \text { value }<0.05 \text { in the univariable Cox regression } \\
\text { analysis. }\end{array}$} \\
\hline \multicolumn{5}{|c|}{$\begin{array}{l}\text { Abbreviations: } \mathrm{HR} \text {, hazard ratio; } \mathrm{CI} \text {, confidence interval; NS, no surgery; SPM, } \\
\text { surgery to both primary and metastatic tumor; GO, gastrectomy only; MO, } \\
\text { metastasectomy only. }\end{array}$} \\
\hline
\end{tabular}

\subsection{Sensitivity analyses}

A total of 5347 patients were enrolled in the sensitivity analyses, and the demographic and clinicopathological characteristics were still different between groups (Supplementary Table 2). Survival analyses found that mOS of SPM (13 months vs. 8 months; $P<0.001)$ and GO (12 months vs. 8 months; $P<0.001)$ patients were both significantly higher than that of NS patients, and MO was not (8 months vs. 8 months; $P=0.893$ ). SPM and GO groups showed comparable survival $(P=0.748)$ and both were significantly better than MO patients $(P<0.001)$. After matching by PSM and CEM, similar results were obtained. Table 4 illustrates death risks of surgery strategies over NS among patients receiving chemotherapy, that is SPM and GO rather than MO were proven to be favorable for survival over non-surgical treatment, even after matching by PSM or CEM. These consistent results implied that SPM and GO could also improve survival from MO or NS in patients who received chemotherapy.

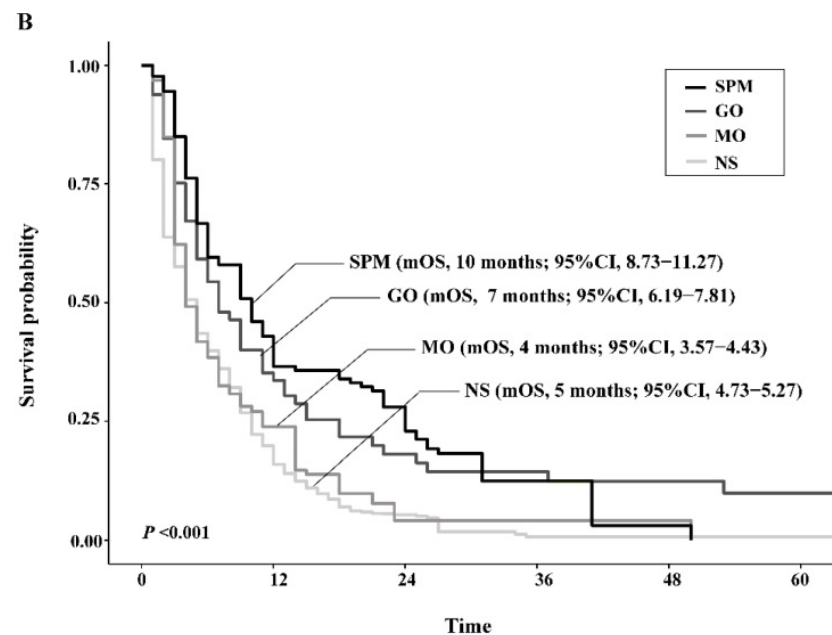

Figure 4. Kaplan-Meier survival curves of patients stratified according to their surgery strategies after propensity score matching (A) or coarsened exact matching (B). Abbreviations: SPM, surgeries to both primary and metastatic tumors; GO, gastrectomy only; MO, metastasectomy only; NS, no surgery; mOS, median overall survival. 
chemotherapy is the mainstay of treatment for mGC patients, we carried out sensitivity analyses after excluding patients receiving no/unknown chemotherapy and obtained consistent results. To the best of our knowledge, this is the first study to demonstrate and compare survival benefits of palliative surgery strategies including gastrectomy and/or metastasectomy with PSM and CEM methods.

Results of the study cast light on the therapeutic role of palliative surgery strategies. Regional resection used to be applied for radical purpose, and it could be achieved in selected potentially resectable mGC patients after conversion chemotherapy with significant survival benefit. [13, 14, 32-36] However, since distal metastasis was a systemic manifestation, the complete resection of primary and metastatic tumors with proved survival benefit might actually be palliative as with a recurrence rate of more than $50 \%$. $[35,37]$ Palliative gastrectomy with or without metastasectomy improved survival probably due to the debulking or cytoreductive effect at its source. Relieving symptoms caused by either the primary or metastatic tumors might as well positively influence mGC patients. However, metastasectomy seemed to obtain little additional survival improvement in the study, which might be attributed to heterogeneity of metastatic loci status of the study population. One research held the view that only metastasectomy could distinguish second primary tumors from metastasis. [29] Thus, MO is not recommended for general application and might be a choice among cautiously selected patients, and SPM could be adopted after adequate cost-and-effectiveness weighing for its numerically better OS compared with GO.

The study has several limitations. First, data on patient comorbidities and performance status, postoperative mortality and morbidity, quality of life, and detailed chemotherapy information are unavailable. Further studies are needed to explore the extra influence of these variables on survival and to select suitable surgery candidates. Second, the circumstances under which surgeries were conducted, like the surgeries are emergent or not and the patients are asymptomatic or not, were not specified in the SEER database. Such information could had better distinguished surgeries as a treatment alternative from emergency operations. Third, PSM and CEM analysis only deal with observable factors, thus other unobservable factors might be confounding and unmatched. Also, part of information is lost during the process of matching. Combination of the results of the two matching methods and multivariate Cox regression analysis in the present study is a dependable solution. Despite these limitations, given the large sample size and the population-based nature of the SEER database, we are able to perform adequately powerful survival analyses.

\section{Conclusions}

Poor prognosis of metastatic gastric cancer promotes oncologists to spare no effort finding and evaluating various treatment strategies for survival prolongation, and the present study demonstrated gastrectomy plus metastasectomy or gastrectomy alone could be adopted as a choice of improving survival in the U.S population. Metastasectomy alone is not recommended except for highly selected patients.

\section{Abbreviations}

GC: gastric cancer; mGC: metastatic GC; SEER: Surveillance, Epidemiology, and End Results Program; SPM: surgeries to both primary and metastatic tumors; GO: gastrectomy only; MO: metastasectomy only; NS: not surgically operated; PSM: propensity score matching; CEM: coarsened exact matching; MDT: multidisciplinary team.

\section{Supplementary Material}

Supplementary tables.

http://www.jcancer.org/v10p0602s1.pdf

\section{Acknowledgements}

We feel grateful that this study was funded by the Natural Science Foundation of Guangdong Province [grant number 2014A030312015]; Science and Technology Program of Guangdong [grant number 2015B020232008]; Science and Technology Program of Guangzhou [grant number 15570006, 201508020250, 201604020003]. We also hold gratitude towards staff members at the National Cancer Institute and their colleagues across the United States and at the Information Management Services, Inc., who have been involved with the Surveillance, Epidemiology, and End Results (SEER) Program.

\section{Competing Interests}

The authors have declared that no competing interest exists.

\section{References}

1. Siegel R, Ma J, Zou Z, Jemal A. Cancer statistics, 2014. CA: A Cancer Journal for Clinicians. 2014; 64: 9-29.

2. Edwards BK, Noone AM, Mariotto AB, Simard EP, Boscoe FP, Henley SJ, et al. Annual Report to the Nation on the status of cancer, 1975-2010, featuring prevalence of comorbidity and impact on survival among persons with lung, colorectal, breast, or prostate cancer. Cancer. 2014; 120: 1290-314.

3. Shridhar R, Almhanna K, Hoffe SE, Fulp W, Weber J, Chuong MD, et al. Increased survival associated with surgery and radiation therapy in metastatic gastric cancer. Cancer. 2013; 119: 1636-42.

4. Waddell T, Verheij M, Allum W, Cunningham D, Cervantes A, Arnold D. Gastric cancer: ESMO-ESSO-ESTRO clinical practice guidelines for diagnosis, treatment and follow-up. European journal of surgical oncology : the journal 
of the European Society of Surgical Oncology and the British Association of Surgical Oncology. 2014; 40: 584-91.

5. Gold JS, Jaques DP, Bentrem DJ, Shah MA, Tang LH, Brennan MF, et al. Outcome of patients with known metastatic gastric cancer undergoing resection with therapeutic intent. Annals of surgical oncology. 2007; 14: 365-72.

6. Fujitani K, Yang HK, Mizusawa J, Kim YW, Terashima M, Han SU, et al. Gastrectomy plus chemotherapy versus chemotherapy alone for advanced gastric cancer with a single non-curable factor (REGATTA): a phase 3, randomised controlled trial. The Lancet Oncology. 2016; 17: 309-18.

7. He MM, Zhang DS, Wang F, Wang ZQ, Luo HY, Jin Y, et al. The role of non-curative surgery in incurable, asymptomatic advanced gastric cancer. PloS one. 2013; 8: e83921.

8. Kim KH, Lee KW, Baek SK, Chang HJ, Kim YJ, Park DJ, et al. Survival benefit of gastrectomy $+/$ - metastasectomy in patients with metastatic gastric cancer receiving chemotherapy. Gastric cancer : official journal of the International Gastric Cancer Association and the Japanese Gastric Cancer Association. 2011; 14: $130-8$

9. Yang SW, Kim MG, Lee JH, Kwon SJ. Role of metastasectomy on overall survival of patients with metastatic gastric cancer. Journal of gastric cancer. 2013; 13: 226-31.

10. Hsu JT, Liao JA, Chuang HC, Chen TD, Chen TH, Kuo CJ, et al. Palliative gastrectomy is beneficial in selected cases of metastatic gastric cancer. BMC palliative care. 2017; 16: 19

11. Chen J, Kong Y, Weng S, Dong C, Zhu L, Yang Z, et al. Outcomes of surgery for gastric cancer with distant metastases: a retrospective study from the SEER database. Oncotarget. 2017; 8: 4342-51

12. Qiu JL, Deng MG, Li W, Zou RH, Li BK, Zheng Y, et al. Hepatic resection for synchronous hepatic metastasis from gastric cancer. European journal of surgical oncology : the journal of the European Society of Surgical Oncology and the British Association of Surgical Oncology. 2013; 39: 694-700.

13. Dittmar Y, Altendorf-Hofmann A, Rauchfuss F, Gotz M, Scheuerlein H, Jandt $\mathrm{K}$, et al. Resection of liver metastases is beneficial in patients with gastric cancer: report on 15 cases and review of literature. Gastric cancer : official journal of the International Gastric Cancer Association and the Japanese Gastric Cancer Association. 2012; 15: 131-6.

14. Iijima $Y$, Akiyama H, Atari M, Fukuhara M, Nakajima $Y$, Kinosita H, et al. Pulmonary Resection for Metastatic Gastric Cancer. Annals of thoracic and cardiovascular surgery: official journal of the Association of Thoracic and Cardiovascular Surgeons of Asia. 2016; 22: 230-6.

15. Rudloff U, Langan RC, Mullinax JE, Beane JD, Steinberg SM, Beresnev T, et al. Impact of maximal cytoreductive surgery plus regional heated intraperitoneal chemotherapy (HIPEC) on outcome of patients with peritoneal carcinomatosis of gastric origin: results of the GYMSSA trial. Journal of surgical oncology. 2014; 110: 275-84.

16. Randolph JJ, Falbe K, Manuel AK \& Balloun JL. A Step-by Step Guide to Propensity Score Matching in R. Practical Assessment, Research \& Evaluation. 2014; 19.

17. Haider AH, David JS, Zafar SN, Gueugniaud PY, Efron DT, Floccard B, et al. Comparative effectiveness of inhospital trauma resuscitation at a French trauma center and matched patients treated in the United States. Annals of surgery. 2013; 258: 178-83.

18. Iacus SM, King G, Porro G. Causal Inference without Balance Checking: Coarsened Exact Matching. Polit Anal. 2012; 20: 1-24.

19. Sutter R, De Marchis GM, Semmlack S, Fuhr P, Ruegg S, Marsch S, et al. Anesthetics and Outcome in Status Epilepticus: A Matched Two-Center Cohort Study. CNS drugs. 2017; 31: 65-74.

20. Izuishi K, Mori H. Recent Strategies for Treating Stage IV Gastric Cancer: Roles of Palliative Gastrectomy, Chemotherapy, and Radiotherapy. Journal of gastrointestinal and liver diseases : JGLD. 2016; 25: 87-94.

21. Lasithiotakis K, Antoniou SA, Antoniou GA, Kaklamanos I, Zoras O. Gastrectomy for stage IV gastric cancer. a systematic review and meta-analysis. Anticancer research. 2014; 34: 2079-85.

22. Sano T, Sasako M, Yamamoto S, Nashimoto A, Kurita A, Hiratsuka M, et al. Gastric cancer surgery: morbidity and mortality results from a prospective randomized controlled trial comparing D2 and extended para-aortic lymphadenectomy--Japan Clinical Oncology Group study 9501. Journal of clinical oncology : official journal of the American Society of Clinical Oncology. 2004; 22: 2767-73.

23. Yoon SS, Yang HK. Lymphadenectomy for gastric adenocarcinoma: should west meet east? The oncologist. 2009; 14: 871-82.

24. Davies AR, Deans DA, Penman I, Plevris JN, Fletcher J, Wall L, et al. The multidisciplinary team meeting improves staging accuracy and treatment selection for gastro-esophageal cancer. Diseases of the esophagus : official journal of the International Society for Diseases of the Esophagus. 2006; 19: 496-503.

25. Pillay B, Wootten AC, Crowe H, Corcoran N, Tran B, Bowden P, et al. The impact of multidisciplinary team meetings on patient assessment, management and outcomes in oncology settings: A systematic review of the literature. Cancer treatment reviews. 2016; 42: 56-72.

26. Warschkow R, Baechtold M, Leung K, Schmied BM, Nussbaum DP, Gloor B, et al. Selective survival advantage associated with primary tumor resection for metastatic gastric cancer in a Western population. Gastric cancer : official journal of the International Gastric Cancer Association and the Japanese Gastric Cancer Association. 2018; 21: 324-37.
27. Gadde R, Tamariz L, Hanna M, Avisar E, Livingstone A, Franceschi D, et al. Metastatic gastric cancer (MGC) patients: Can we improve survival by metastasectomy? A systematic review and meta-analysis. Journal of surgical oncology. 2015; 112: 38-45.

28. Markar SR, Mikhail S, Malietzis G, Athanasiou T, Mariette C, Sasako M, et al. Influence of Surgical Resection of Hepatic Metastases From Gastric Adenocarcinoma on Long-term Survival: Systematic Review and Pooled Analysis. Annals of surgery. 2016; 263: 1092-101.

29. Nakamura T, Homma Y, Miyata N, Ushida S, Suzuki K, Otsuki Y, et al. Only surgical resection can identify the second primary lung cancer out of the metastasis after gastric cancer surgery. Japanese journal of clinical oncology. 2012; 42: 609-11.

30. Petrelli F, Coinu A, Cabiddu M, Ghilardi M, Borgonovo K, Lonati V, et al. Hepatic resection for gastric cancer liver metastases: A systematic review and meta-analysis. Journal of surgical oncology. 2015; 111: 1021-7.

31. Shiono S, Sato T, Horio H, Chida M, Matsuguma H, Ozeki Y, et al. Outcomes and prognostic factors of survival after pulmonary resection for metastatic gastric cancer. European journal of cardio-thoracic surgery : official journal of the European Association for Cardio-thoracic Surgery. 2013; 43: e13-6.

32. Wang W, Liang H, Zhang H, Wang X, Xue Q, Zhang R. Prognostic significance of radical surgical treatment for gastric cancer patients with synchronous liver metastases. Medical oncology (Northwood, London, England). 2014; 31: 258.

33. Ito S, Oki E, Nakashima Y, Ando K, Hiyoshi Y, Ohgaki K, et al. Clinical significance of adjuvant surgery following chemotherapy for patients with initially unresectable stage IV gastric cancer. Anticancer research. 2015; 35: 401-6.

34. Al-Batran SE, Homann N, Pauligk C, Illerhaus G, Martens UM, Stoehlmacher J, et al. Effect of Neoadjuvant Chemotherapy Followed by Surgical Resection on Survival in Patients With Limited Metastatic Gastric or Gastroesophageal Junction Cancer: The AIO-FLOT3 Trial. JAMA oncology. 2017; 3: 1237-44.

35. Schmidt T, Alldinger I, Blank S, Klose J, Springfeld C, Dreikhausen L, et al. Surgery in oesophago-gastric cancer with metastatic disease: Treatment, prognosis and preoperative patient selection. European journal of surgical oncology : the journal of the European Society of Surgical Oncology and the British Association of Surgical Oncology. 2015; 41: 1340-7.

36. Polkowska-Pruszynska B, Rawicz-Pruszynski K, Cisel B, Sitarz R, Polkowska G, Krupski W, et al. Liver metastases from gastric carcinoma: A Case report and review of the literature. Current problems in cancer. 2017; 41: 222-30.

37. Tatsubayashi T, Tanizawa Y, Miki Y, Tokunaga M, Bando E, Kawamura T, et al. Treatment outcomes of hepatectomy for liver metastases of gastric cancer diagnosed using contrast-enhanced magnetic resonance imaging. Gastric cancer : official journal of the International Gastric Cancer Association and the Japanese Gastric Cancer Association. 2017; 20: 387-93. 